\title{
Universal structure of the Drell-Yan process beyond threshold
}

\author{
Domenico Bonocore ${ }^{* \dagger}$ \\ Nikhef \\ E-mail: d.bonocore@nikhef.nl
}

\begin{abstract}
We review recent results in the investigation of threshold logarithms at next-to-leading power considering the case of the Drell-Yan cross section at NNLO. We first show how they can be reproduced with a method of region calculation. Then we move to an approach based on softcollinear factorization, showing that the entire logarithmic structure can be reproduced by means of universal functions.
\end{abstract}

12th International Symposium on Radiative Corrections (Radcor 2015) and LoopFest XIV (Radiative Corrections for the LHC and Future Colliders)

15-19 June, 2015

UCLA Department of Physics \& Astronomy Los Angeles, USA

\footnotetext{
* Speaker.

${ }^{\dagger}$ Talk based on a collaboration with E. Laenen, L. Magnea, S. Melville, L. Vernazza and C.D. White.
} 


\section{Introduction}

It is well known that cross sections close to the threshold are plagued by potentially large logarithms of soft and collinear origin that often need to be resummed to all orders in order to maintain the predictability of perturbation theory. For a variety of processes the logarithmic structure of these cross sections follows the pattern

$$
\frac{d \sigma}{d \xi}=\sum_{n=0}^{\infty} \alpha_{s}^{n} \sum_{m=0}^{2 n-1}\left[a_{n m}\left(\frac{\log ^{m}(\xi)}{\xi}\right)_{+}+b_{n m} \log ^{m}(\xi)+\mathscr{O}(\xi)\right]
$$

where $\xi$ is a dimensionless variable that vanishes in the threshold limit.

The literature about the resummation of the leading terms, which are plus-distributions in momentum space, is extensive [1-3] and relies largely on the eikonal approximation. Preliminary studies on the structure of the subleading terms have been performed in [4-11]. They typically require a generalization of standard techniques to a subleading degree of approximation (known in the literature as "next-to-soft" or "next-to-eikonal"). However, a general formalism that resums such contributions is still lacking. In parallel, the subject has been investigated at amplitude level in more formal contexts [12-21] and with the use of soft-collinear effective theory [22]. Here we report recent results that shed light on this matter [23,24], focusing on the specific case of the Drell-Yan process.

This process, at parton level, describes the production of an off-shell gauge boson of invariant mass $Q^{2}$ from a quark-antiquark pair of invariant mass $s$. Introducing the dimensionless ratio $z=Q^{2} / s$, the threshold limit is achieved in the limit $z \rightarrow 1$. Among all the reasons that make this process interesting, probably the most relevant one for LHC physics is the similarity with Higgs boson production through gluon-fusion, thanks to a common topology of the diagrams involved [25-27]. However, there is also a more technical reason for studying it: in this process kinematical constraints force threshold radiation to be soft and thus the threshold limit corresponds to the vanishing of the energy of the real gluon. However, as we shall see, this does not imply that threshold logarithms are insensitive to the virtual gluon when this is collinear.

The case considered here is the $C_{F}^{2}$ part of the real-virtual interference at NNLO [28], whose diagrams are represented in Figure 1. While the restriction to the abelian part is merely dictated by simplicity, in order to minimize technical complications, the choice of this set of diagrams is related to the difference between soft (or eikonal) and threshold expansion. These two do not coincide when there are both real and virtual gluons since the threshold expansion also includes collinear effects. Therefore we cannot use factorization techniques based on effective next-to-soft vertices that turned out to be successful for diagrams with two real gluons [6].

To investigate these issues, we consider the contribution of these diagrams to the NNLO $K$ factor, which is defined as

$$
K^{(2)}(z)=\frac{1}{\sigma_{B}} \frac{d \sigma^{(2)}}{d Q^{2}}
$$

where $\sigma_{B}$ is the Born cross section and the superscript stands for NNLO in $\alpha_{s}$. In [23] the exact result expanded at subleading order in the threshold was presented. Discarding for simplicity 


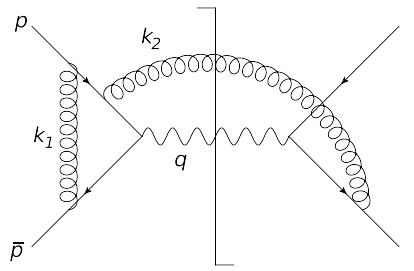

(a)

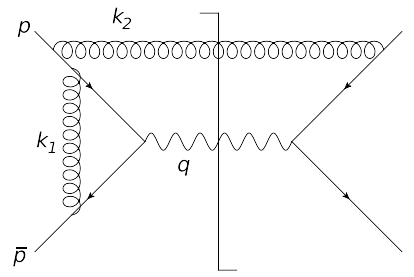

(d)

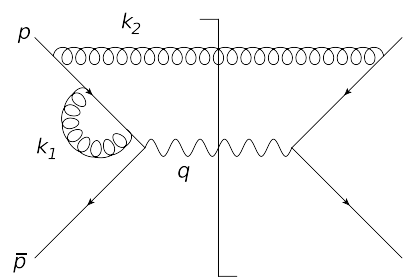

(g)

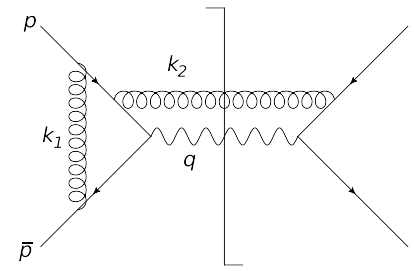

(b)

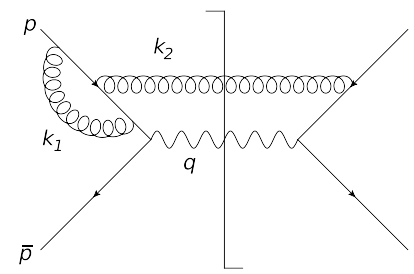

(e)

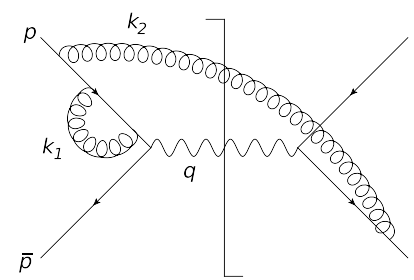

(h)

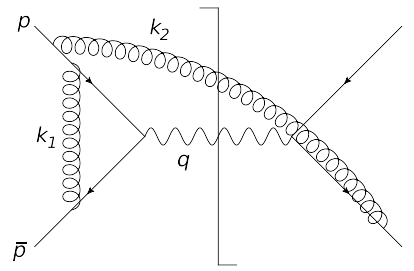

(c)

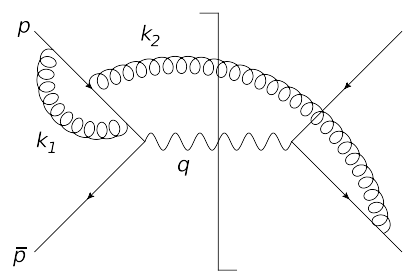

$(f)$

Figure 1: Abelian-like diagrams for the real-virtual interference of NNLO Drell-Yan production. Diagrams obtained by complex conjugation and $p \leftrightarrow \bar{p}$ are omitted.

transcendental constants, it reads

$$
\begin{aligned}
K^{(2)}(z)=\left(\frac{\alpha_{s}}{4 \pi} C_{F}\right)^{2} & {\left[\frac{32 \mathscr{D}_{0}(z)-32}{\varepsilon^{3}}+\frac{-64 \mathscr{D}_{1}(z)+48 \mathscr{D}_{0}(z)+64 L(z)-96}{\varepsilon^{2}}\right.} \\
& +\frac{64 \mathscr{D}_{2}(z)-96 \mathscr{D}_{1}(z)+128 \mathscr{D}_{0}(z)-64 L^{2}(z)+208 L(z)-196}{\varepsilon} \\
& -\frac{128 \mathscr{D}_{3}(z)}{3}+96 \mathscr{D}_{2}(z)-256 \mathscr{D}_{1}(z)+256 \mathscr{D}_{0}(z) \\
& \left.+\frac{128}{3} L^{3}(z)-232 L^{2}(z)+412 L(z)-408+\mathscr{O}(1-z)\right]
\end{aligned}
$$

where

$$
\mathscr{D}_{m}(\xi)=\left(\frac{\log ^{m}(\xi)}{\xi}\right)_{+} \quad \text { and } \quad L(z)=\log (1-z)
$$

In order to understand the underlying singular (universal) nature of these logarithms, in the following sections we review how to reproduce their coefficients with two approaches: the method of regions and a factorization approach. 


\section{Analysis with the method of regions}

The method of regions [29] is a powerful tool to compute loop integrals in a specific asymptotic limit and amounts to expanding the integrand in various regions according to its singular behavior. In the case we want to consider, the small parameter $\lambda$ of this asymptotic limit defines the soft (or eikonal) expansion and is proportional to the square root of the energy of the real gluon. Since we want to perform such an expansion at subleading order [23], we distinguish between the leading eikonal (E) and subleading next-to-eikonal (NE) contributions. The relevant regions are defined by the momentum $\ell$ of the virtual gluon, according to the different scaling of its components, which in light-cone coordinates reads

$$
\begin{aligned}
\text { hard }: \ell_{h} & \sim(1,1,1) & \text { collinear }: \ell_{c} & \sim\left(1, \lambda, \lambda^{2}\right) \\
\text { soft }: \ell_{s} & \sim\left(\lambda^{2}, \lambda^{2}, \lambda^{2}\right) & \text { anticollinear }: \ell_{\bar{c}} & \sim\left(\lambda^{2}, \lambda, 1\right) .
\end{aligned}
$$

However, most of the regions are actually zero for some subsets of diagrams. First of all, in the soft region all integrals are scaleless and thus must be set to zero ${ }^{1}$. Then, at $\mathrm{E}$ level, (anti)collinear regions are non-zero on a diagram by diagram basis. However, in Feynman gauge ${ }^{2}$, they cancel in every pair of graphs in which the attachments of the two gluons are swapped. Finally, self-energy diagrams (e)-(h) are made out of pure collinear regions. In conclusion, non-vanishing contributions come from the hard region $K_{h}^{(2)}$ of diagrams (a)-(d) (both at $\mathrm{E}$ and NE level) and from the collinear region $K_{c+\bar{c}}^{(2)}$ of all diagrams (only at NE level). One finds

$$
\begin{aligned}
& K_{\mathrm{E}, \mathrm{h}}^{(2)}(z)=\left(\frac{\alpha_{s}}{4 \pi} C_{F}\right)^{2}\left[\frac{32 \mathscr{D}_{0}(z)}{\varepsilon^{3}}+\frac{-64+48 \mathscr{D}_{0}(z)-64 \mathscr{D}_{1}(z)}{\varepsilon^{2}}\right. \\
& +\frac{-96+128 \mathscr{D}_{0}(z)-96 \mathscr{D}_{1}(z)+64 \mathscr{D}_{2}(z)+128 L(z)}{\varepsilon} \\
& +256 \mathscr{D}_{0}(z)-256 \mathscr{D}_{1}(z)+96 \mathscr{D}_{2}(z)-\frac{128 \mathscr{D}_{3}(z)}{3} \\
& \left.+192 L(z)-128 L^{2}(z)-256\right] \text {, } \\
& K_{\mathrm{NE}, \mathrm{h}}^{(2)}(z)=\left(\frac{\alpha_{s}}{4 \pi} C_{F}\right)^{2}\left[-\frac{32}{\varepsilon^{3}}+\frac{16+64 L(z)}{\varepsilon^{2}}+\frac{-80+32 L(z)-64 L^{2}(z)}{\varepsilon}\right. \\
& \left.+160 L(z)-32 L^{2}(z)+\frac{128}{3} L^{3}(z)-128\right], \\
& K_{\mathrm{NE}, \mathrm{c}+\overline{\mathrm{c}}}^{(2),(\mathrm{d})}(z)=\left(\frac{\alpha_{s}}{4 \pi} C_{F}\right)^{2}\left[-\frac{8}{\varepsilon^{2}}+\frac{24 L(z)}{\varepsilon}-36 L^{2}(z)+16\right], \\
& K_{\mathrm{NE}, \mathrm{c}+\overline{\mathrm{c}}}^{(2),(\mathrm{h})}(z)=\left(\frac{\alpha_{s}}{4 \pi} C_{F}\right)^{2}\left[-\frac{8}{\varepsilon^{2}}+\frac{-20+24 L(z)}{\varepsilon}+60 L(z)-36 L^{2}(z)-40\right] .
\end{aligned}
$$

${ }^{1}$ Setting to zero scaleless integrals in dimensional regularization is one of the prescriptions of the method of regions [30]. Note that the soft region is different from the traditional definition of the soft function: this one is also represented by scaleless integrals but, being defined as the VEV of composite operators, needs a further UV renormalization and thus it is not zero.

${ }^{2}$ This choice is consistent with the analysis carried out with a factorization approach. 
The sum of these four contributions reproduces the full result of Eq. (1.3). Plus-distributions are exclusively captured by the hard function and, as expected, they are entirely reproduced already at leading order (E) in the soft expansion. However, the presence of $L(z)$ 's in Eq. (2.2), which are subleading in $(1-z)$, shows explicitly the difference between threshold and eikonal (or soft) expansion, and in this case it is due to corrections to the phase space measure.

We can investigate further the role of these regions in the context of a naïve factorization approach. Let us consider the real emission of a next-to-soft gluon of momentum $k$ from a hard line of momentum $p_{i}$. Let us assume further that this on-shell gluon factorizes from the hard line and therefore the entire dependence on $k$ can be represented by a universal factor $V[4,5,18]$ that can be expanded at $\mathrm{E}$ and $\mathrm{NE}$ level and reads respectively

$$
\begin{aligned}
V_{E} & =-\frac{p_{i}^{\mu}}{p_{i} \cdot k} \\
V_{N E} & =\frac{k^{\mu}}{2 p_{i} \cdot k}-\frac{i k_{v} \Sigma^{\mu v}}{p_{i} \cdot k} .
\end{aligned}
$$

From these it is clear that eikonal interactions, as it is well known, cannot resolve the spin of the emitting particle (and therefore they are represented by a scalar vertex). NE emissions instead are spin-dependent and contain the Lorentz generator $\Sigma^{\mu v}=\frac{i}{4}\left[\gamma^{\mu}, \gamma^{v}\right]$.

Working in a naïve factorization framework, we can use (2.6)-(2.7) to compute the contribution to the NNLO $K$-factor shown in Fig. (1). In particular, diagrams can be written as a one-loop amplitude (either a vertex form factor or a self energy correction) times the real emission at $\mathrm{E}$ or NE level. However, following this procedure, most of the diagrams vanish, either because after taking the spinor trace we encounter $p_{i}^{2}=0$ or because the self energy diagram itself is zero. The only non vanishing ones would be diagrams (a) and (c), whose sum matches with the result from an exact computation. Hence, all plus-distributions and the leading logarithms are reproduced by this naïve factorizaion approach. On the other hand we know from the method of region analysis that the entire hard region is given by diagram (a)-(d). Therefore, recalling that diagram (d) is zero [24], we conclude that the contributions which are left out in this naïve factorization approach are the entire collinear region and the hard region of diagram (b).

In conclusion, with the method of regions we have been able to reproduce the entire logarithmic structure, attributing each logarithm to a specific singular region. Moreover, it has been possible to identify the kinematical nature of the terms that break a naïve factorization approach. However, if we want to address an all-order interpretation for the entire logarithmic structure, we need to revisit our factorization approach.

\section{A new factorization approach}

The starting point is the soft-collinear factorization formula for scattering amplitudes in covariant gauge [31]. For the quark form factor $\mathscr{A}$ of external momenta $p_{1}$ and $p_{2}$ it reads

$$
\begin{aligned}
& \mathscr{A}\left(\frac{p_{1} \cdot p_{2}}{\mu^{2}}, \alpha_{s}\left(\mu^{2}\right), \varepsilon\right)=\mathscr{H}(\left.\left\{p_{i}\right\},\left\{n_{i}\right\}, \alpha_{s}\left(\mu^{2}\right), \varepsilon\right) \times \overline{\mathscr{S}}\left(\left\{\beta_{i}\right\},\left\{n_{i}\right\}, \alpha_{s}\left(\mu^{2}\right), \varepsilon\right) \\
& \times \prod_{i=1}^{2} J_{i}\left(p_{i}, n_{i}, \alpha_{s}\left(\mu^{2}\right), \varepsilon\right)
\end{aligned}
$$


where $\mathscr{H}, J$ and $\overline{\mathscr{S}}$ are respectively the hard, jet and reduced soft functions. Their definitions in terms of composite operators can be found e.g. in [31,32], where also an explicit one loop expression for them is given. For each external leg $i, n_{i}$ is an auxiliary factorization vector, used to define the direction of the Wilson lines that appear in the operator definition. In order to make a closer connection with the method of regions and to simplify the calculation, in the following it will be assumed that $n_{i}^{2}=0$.

Building upon the work done in [33], in [24] this formula has been generalized to the case of an amplitude $\mathscr{A}^{\mu}$ radiating an extra (next-to-)soft gluon of momentum $k^{\mu}$

$$
\begin{aligned}
\mathscr{A}^{\mu}\left(p_{j}, k\right) & =\sum_{i=1}^{2}\left[q_{i}\left(\frac{\left(2 p_{i}-k\right)^{\mu}}{2 p_{i} \cdot k-k^{2}}+G_{i}^{v \mu} \frac{\partial}{\partial p_{i}^{v}}\right) \mathscr{A}\left(p_{i} ; p_{j}\right)\right. \\
& \left.+\mathscr{H}\left(p_{j}, n_{j}\right) \overline{\mathscr{S}}\left(\beta_{j}, n_{j}\right) G_{i}^{\nu \mu}\left(J_{v}\left(p_{i}, k, n_{i}\right)-q_{i} \frac{\partial}{\partial p_{i}^{v}} J\left(p_{i}, n_{i}\right)\right) \prod_{j \neq i} J\left(p_{j}, n_{j}\right)\right],
\end{aligned}
$$

where the tensor $G^{\mu v}$ is defined in terms of the Minkowski metric $\eta^{\mu v}$ as

$$
G^{\mu v}=\eta^{\mu v}-\frac{(2 p-k)^{v}}{2 p \cdot k-k^{2}} k^{\mu}
$$

This formula shows, up to next-to-soft level, how a radiative amplitude $\mathscr{A}^{\mu}$ is related to the nonradiative one $\mathscr{A}$. The first term in the first line of (3.2) represents the scalar contribution to a factorizable external emission, already discussed in equations (2.6)-(2.7). At eikonal level, this is the only term which survives and simply describes traditional factorization via an external eikonal emission. Then there are two derivative contributions (acting respectively on the full form factor and on the jet function) which were present in the original Low-Burnett-Kroll analysis [34, 35]. The last non-derivative term was first discussed by Del Duca [33] and requires the definition of the radiative jet function. Physically, it represents a soft emission from the jet function and it is defined as

$$
J_{\mu}\left(p, n, k, \alpha_{s}\left(\mu^{2}\right), \varepsilon\right) u(p)=\int d^{d} y \mathrm{e}^{-\mathrm{i}(p-k) \cdot y}\left\langle 0\left|\Phi_{n}(y, \infty) \psi(y) j_{\mu}(0)\right| p\right\rangle,
$$

While this formal definition has been known for a long time, its explicit expression was computed only in [24]. For $n_{i}^{2}=0$ it reads respectively at tree level and one loop

$$
\begin{aligned}
J^{v(0)}(p, n, k) & =-\frac{p^{v}}{p \cdot k}+\frac{k^{v}}{2 p \cdot k}-\frac{\mathrm{i} k_{\alpha} \Sigma^{\alpha \mu}}{2 p \cdot k} \\
J^{v(1)}(p, n, k ; \varepsilon) & =(2 p \cdot k)^{-\varepsilon}\left[\left(\frac{2}{\varepsilon}+4+8 \varepsilon\right)\left(\frac{n \cdot k}{p \cdot k} \frac{p^{v}}{p \cdot n}-\frac{n^{v}}{p \cdot n}\right)-(1+2 \varepsilon) \frac{\mathrm{i} k_{\alpha} \Sigma^{\alpha v}}{p \cdot k}\right. \\
& \left.+\left(\frac{1}{\varepsilon}-\frac{1}{2}-3 \varepsilon\right) \frac{k^{v}}{p \cdot k}+(1+3 \varepsilon)\left(\frac{\gamma^{v} \not h}{p \cdot n}-\frac{p^{v} \not k \not h}{p \cdot k p \cdot n}\right)\right]+\mathscr{O}\left(\varepsilon^{2}, k\right) .
\end{aligned}
$$

We observe that the tree level contribution is equal to the sum of equation (2.6) and (2.7), and therefore describes a full spin-dependent factorizable emission. The one loop expression instead is proportional to $(2 p \cdot k)^{-\varepsilon}$, which is the typical collinear scaling. Moreover we note that at one loop 
the spin dependent part is subleading in $\varepsilon$ and therefore its contribution will affect only subleading logarithms of the $K$-factor.

A number of simplifications can be done. Following a renormalization group argument, it is possible to perform a bare calculation ignoring UV counterterms. This simplifies considerably the factorization formula: without UV counterterms, for $n_{i}^{2}=0$, the jet function becomes simply equal to the identity since its radiative corrections vanish. Then, in order to make a more direct connection with the method of region analysis, we can set $n_{i}$ to be equal to the anti-collinear direction of the leg $i$ (i.e. $n_{1}=p_{2}$ and $n_{2}=p_{1}$ ). This is physically motivated by the fact that the vector $n_{i}$ has been introduced via the Wilson line as a replacement for the parton of momentum $p_{i}$. Finally, we can expand the factorization formula at one loop and make use of (3.5). Thus we get

$$
\begin{gathered}
\mathscr{A}^{\mu,(1)}\left(p_{j}, k\right)=\sum_{i=1}^{2}\left[\left(\frac{p_{i}^{\mu}}{p_{i} \cdot k_{2}}-\frac{\not k_{2} \gamma^{\mu}}{2 p_{i} \cdot k_{2}}\right) \mathscr{A}^{(1)}\left(p_{i} ; p_{j}\right)+\left(G_{i}^{v \mu} \frac{\partial}{\partial p_{i}^{\nu}}\right) \mathscr{A}^{(1)}\left(p_{i} ; p_{j}\right)\right. \\
\left.+J^{\mu(1)}\left(p_{i}, k\right) \mathscr{A}^{(0)}\left(p_{i} ; p_{j}\right)\right] .
\end{gathered}
$$

This is the final formula we want to use to reconstruct the NNLO $K$-factor. It is made out of three main contributions:

- a factorized emission external to the one loop form factor,

- a derivative of the one loop form factor,

- the one loop radiative jet.

In the following we present the contribution to the $K$-factor separately for these three contributions, comparing them with the method of regions analysis, and showing that the entire logarithmic structure of Eq. (1.3) is reproduced.

\subsection{External contribution}

The set of logarithms coming from the form factor dressed by an external emission reads

$$
\begin{aligned}
K_{\mathrm{ext}}^{(2)}(z)=\left(\frac{\alpha_{s}}{4 \pi} C_{F}\right)^{2} & \left\{\frac{32}{\varepsilon^{3}}\left[\mathscr{D}_{0}(z)-1\right]+\frac{8}{\varepsilon^{2}}\left[-8 \mathscr{D}_{1}(z)+6 \mathscr{D}_{0}(z)+8 L(z)-14\right]\right. \\
& +\frac{16}{\varepsilon}\left[4 \mathscr{D}_{2}(z)-6 \mathscr{D}_{1}(z)+8 \mathscr{D}_{0}(z)-4 L^{2}(z)+14 L(z)-14\right] \\
& -\frac{128}{3} \mathscr{D}_{3}(z)+96 \mathscr{D}_{2}(z)-256 \mathscr{D}_{1}(z)+256 \mathscr{D}_{0}(z) \\
& \left.+\frac{128}{3} L^{3}(z)-224 L^{2}(z)+448 L(z)-512\right\} .
\end{aligned}
$$

As expected all $\mathscr{D}$ 's of the total result come from this contribution (i.e. eikonal emissions factorize). This term collects the entire factorizable part of the $K$-factor, which from the method of region analysis we found to be equal to the hard regions of diagrams (a) and (c). 


\subsection{Derivative contribution}

The contribution from the derivative of the full form factor gives

$$
K_{\partial \mathscr{A}}^{(2)}(z)=\left(\frac{\alpha_{s}}{4 \pi} C_{F}\right)^{2}\left\{\frac{32}{\varepsilon^{2}}+\frac{16}{\varepsilon}[-4 L(z)+3]+64 L^{2}(z)-96 L(z)+128\right\},
$$

This term is the one related to original analysis of Low, Burnett and Kroll and it matches with the non-factorizable part of the hard region, i.e. the hard region of diagram (b). As expected it contains no $\mathscr{D}$ 's, and thus does not spoil eikonal factorization.

\subsection{The radiative jet contribution}

The third and last contribution comes the radiative jet function. Just as the derivative contribution, it breaks naïve factorization and reads

$$
K_{\mathrm{jet}}^{(2)}(z)=\left(\frac{\alpha_{s}}{4 \pi} C_{F}\right)^{2}\left\{-\frac{16}{\varepsilon^{2}}+\frac{4}{\varepsilon}[12 L(z)-5]-72 L^{2}(z)+60 L(z)-24\right\} .
$$

This term matches precisely the total collinear region, given by the sum of equations (2.4) and (2.5), and it is the source of breakdown of next-to-soft theorems at loop level, as first found by [33].

\section{Conclusions}

We considered the NNLO Drell-Yan $K$-factor as case study for the investigation of threshold logarithms at next-to-leading power. We first showed how this can be reproduced by a method of region calculation, isolating and studying the nature of the terms that break a naive factorization. Then we moved to an approach based on the operator formalism of soft-collinear factorization, showing that again the entire logarithmic structure can be reproduced. Crucial to this analysis was the collinear region and the computation of the radiative jet. Reproducing all threshold logarithms at next-to-leading power via universal functions is a very promising result that should pave the way for a full resummation formalism.

\section{References}

[1] G. F. Sterman, "Summation of Large Corrections to Short Distance Hadronic Cross-Sections," Nucl. Phys. B281 (1987) 310.

[2] S. Catani and L. Trentadue, "Resummation of the QCD Perturbative Series for Hard Processes," Nucl. Phys. B327 (1989) 323.

[3] H. Contopanagos, E. Laenen, and G. F. Sterman, "Sudakov factorization and resummation," Nucl. Phys. B484 (1997) 303-330, arXiv: hep-ph/9604313 [hep-ph] .

[4] E. Laenen, L. Magnea, and G. Stavenga, "On next-to-eikonal corrections to threshold resummation for the Drell-Yan and DIS cross sections," Phys. Lett. B669 (2008) 173-179, arXiv: 0807.4412 [hep-ph] .

[5] E. Laenen, G. Stavenga, and C. D. White, "Path integral approach to eikonal and next-to-eikonal exponentiation," JHEP 0903 (2009) 054, arXiv:0811.2067 [hep-ph] . 
[6] E. Laenen, L. Magnea, G. Stavenga, and C. D. White, "Next-to-eikonal corrections to soft gluon radiation: a diagrammatic approach," JHEP 1101 (2011) 141, arXiv:1010.1860 [hep-ph].

[7] S. Moch and A. Vogt, "On non-singlet physical evolution kernels and large-x coefficient functions in perturbative QCD,” JHEP 11 (2009) 099, arXiv:0909.2124 [hep-ph] .

[8] S. Moch and A. Vogt, "Threshold Resummation of the Structure Function F(L)," JHEP 04 (2009) 081, arXiv:0902.2342 [hep-ph].

[9] G. Grunberg and V. Ravindran, “On threshold resummation beyond leading 1-x order,” JHEP 10 (2009) 055, arXiv:0902.2702 [hep-ph].

[10] L. Apolinário, N. Armesto, J. G. Milhano, and C. A. Salgado, "Medium-induced gluon radiation and colour decoherence beyond the soft approximation," JHEP 02 (2015) 119, arXiv: 1407.0599 [hep-ph].

[11] A. A. Almasy, N. A. Lo Presti, and A. Vogt, "Generalized threshold resummation in inclusive DIS and semi-inclusive electron-positron annihilation," arXiv:1511.08612 [hep-ph].

[12] C. D. White, "Factorization Properties of Soft Graviton Amplitudes," JHEP 05 (2011) 060, arXiv:1103.2981 [hep-th].

[13] F. Cachazo and A. Strominger, "Evidence for a New Soft Graviton Theorem,” arXiv: 1404.4091 [hep-th].

[14] E. Casali, "Soft sub-leading divergences in Yang-Mills amplitudes," JHEP 08 (2014) 077, arXiv:1404.5551 [hep-th].

[15] Z. Bern, S. Davies, and J. Nohle, “On Loop Corrections to Subleading Soft Behavior of Gluons and Gravitons,” Phys. Rev. D90 no. 8, (2014) 085015, arXiv:1405.1015 [hep-th] .

[16] H. Luo, P. Mastrolia, and W. J. Torres Bobadilla, "Subleading soft behavior of QCD amplitudes," Phys. Rev. D91 no. 6, (2015) 065018, arXiv:1411.1669 [hep-th] .

[17] A. J. Larkoski, "Conformal Invariance of the Subleading Soft Theorem in Gauge Theory," Phys. Rev. D90 no. 8, (2014) 087701, arXiv:1405.2346 [hep-th].

[18] C. D. White, "Diagrammatic insights into next-to-soft corrections," Phys. Lett. B737 (2014) 216-222, arXiv:1406.7184 [hep-th].

[19] Z. Bern, S. Davies, P. Di Vecchia, and J. Nohle, "Low-Energy Behavior of Gluons and Gravitons from Gauge Invariance," Phys. Rev. D90 no. 8, (2014) 084035, arXiv:1406.6987 [hep-th] .

[20] J. Broedel, M. de Leeuw, J. Plefka, and M. Rosso, "Constraining subleading soft gluon and graviton theorems," Phys. Rev. D90 no. 6, (2014) 065024, arXiv:1406.6574 [hep-th] .

[21] A. Brandhuber, E. Hughes, B. Spence, and G. Travaglini, “One-Loop Soft Theorems via Dual Superconformal Symmetry," arXiv:1511.06716 [hep-th].

[22] A. J. Larkoski, D. Neill, and I. W. Stewart, "Soft Theorems from Effective Field Theory," JHEP 06 (2015) 077, arXiv:1412.3108 [hep-th] .

[23] D. Bonocore, E. Laenen, L. Magnea, L. Vernazza, and C. D. White, "The method of regions and next-to-soft corrections in Drell-Yan production,” Phys.Lett. B742 (2015) 375-382, arXiv:1410.6406 [hep-ph].

[24] D. Bonocore, E. Laenen, L. Magnea, S. Melville, L. Vernazza, et al., "A factorization approach to next-to-leading-power threshold logarithms,” JHEP 1506 (2015) 008, arXiv: 1503.05156 [hep-ph] . 
[25] C. Anastasiou, C. Duhr, F. Dulat, F. Herzog, and B. Mistlberger, "Higgs Boson Gluon-Fusion Production in QCD at Three Loops," Phys. Rev. Lett. 114 (2015) 212001, arXiv: 1503.06056 [hep-ph].

[26] C. Anastasiou, C. Duhr, F. Dulat, E. Furlan, T. Gehrmann, F. Herzog, and B. Mistlberger, "Higgs boson gluon-fusion production at threshold in $\mathrm{N}^{3}$ LO QCD," Phys. Lett. B737 (2014) 325-328, arXiv:1403.4616 [hep-ph].

[27] Y. Li, A. von Manteuffel, R. M. Schabinger, and H. X. Zhu, "Soft-virtual corrections to Higgs production at N²O," Phys. Rev. D91 (2015) 036008, arXiv:1412.2771 [hep-ph] .

[28] R. Hamberg, W. L. van Neerven, and T. Matsuura, “A Complete calculation of the order $\alpha-s^{2}$ correction to the Drell-Yan K factor,” Nucl. Phys. B359 (1991) 343-405. [Erratum: Nucl. Phys.B644,403(2002)].

[29] M. Beneke and V. A. Smirnov, “Asymptotic expansion of Feynman integrals near threshold," Nucl.Phys. B522 (1998) 321-344, arXiv: hep-ph/9711391 [hep-ph] .

[30] B. Jantzen, "Foundation and generalization of the expansion by regions," JHEP 12 (2011) 076, arXiv:1111.2589 [hep-ph].

[31] L. J. Dixon, L. Magnea, and G. F. Sterman, "Universal structure of subleading infrared poles in gauge theory amplitudes," JHEP 0808 (2008) 022, arXiv:0805.3515 [hep-ph ] .

[32] E. Gardi and L. Magnea, "Factorization constraints for soft anomalous dimensions in QCD scattering amplitudes," JHEP 03 (2009) 079, arXiv:0901.1091 [hep-ph] .

[33] V. Del Duca, "High-energy bremsstrahlung theorems for soft photons," Nucl. Phys. B345 (1990) 369-388.

[34] F. E. Low, "Bremsstrahlung of very low-energy quanta in elementary particle collisions," Phys. Rev. 110 (1958) 974-977.

[35] T. H. Burnett and N. M. Kroll, "Extension of the low soft photon theorem," Phys. Rev. Lett. 20 (1968) 86. 